\title{
1997 WESTERN NEW YORK IMAGE PROCESSING WORKSHOP
}

Friday September 19,1997

\author{
University of Rochester \\ Center for Optoelectronics and Imaging \\ Rochester, New York
}

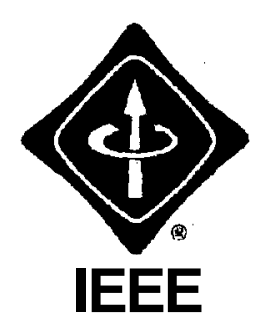

Sponsored by the IEEE Signal Processing Society, Rochester Chapter in Cooperation with the

Center for Electronic Imaging Systems, University of Rochester 


\section{WELCOME AND INTRODUCTION}

On behalf of the workshop organizing committee I welcome you to the 1997 Western New York Image Processing Workshop. This is the first workshop sponsored by the Rochester Chapter of the IEEE Signal Processing Society, and naturally it focuses on digital image processing and its applications. It is intended to provide an opportunity for personal interaction and exchange of ideas between researchers in and around Western New York. Rochester, the home of the Eastman Kodak Company and the Xerox Corporation, has a long and recognized tradition in imaging and provides the ideal setting for such an event. Academic programs with emphasis in digital imaging have flourished at the University of Rochester and the Rochester Institute of Technology, and cooperative efforts between academia and industry have been established through the Center for Electronic Imaging Systems and other programs.

The workshop consists of a single track of presentations grouped into four sessions, with the subject matter kept as general as possible to encourage participation. The presenters represent six academic institutions and two companies, and the number of participants is expected to be well over seventy. Based on this positive response, I believe that similar workshops will continue to take place in years to come with adjusted format and focus to best serve the evolving needs of the research community.

I would like to acknowledge a number of people for their contributions. First, I would like to thank Dr. Michael Kriss and Ms. Janet Eckman from the Center of Electronic Imaging Systems for their help in reserving the Center for Optoelectronics and Imaging. I would also like to express my gratitude to the IEEE Rochester Section Officers for their support, particularly Mr. James Ziobro, the IEEE Rochester Section Secretary, for his continued participation in the Signal Processing Society activities. Most of all I would like to thank the members of the organizing committee, Dr. Gozde Bozdagi, Dr. Murat Tekalp, Dr. Ricardo deQueiroz, Mr. George Sotak, and Dr. Eli Saber, for their hard work and dedication. Without them this workshop would not have been possible.

Enjoy the workshop and keep on processing!

Andreas E. Savakis

General Chair 


\section{WORKSHOP COMMITTEE}

\section{General Chair}

Dr. Andreas E. Savakis

Eastman Kodak Company

Rochester, New York 14653

savakis@kodak.com

\section{Technical Committee}

Dr. Gozde Bozdagi

Xerox Corporation

Webster, New York 14580

bozdagi@wrc.xerox.com

Dr. A. Murat Tekalp

University of Rochester

Rochester, New York 14627

tekalp@ee.rochester.edu

Dr. Ricardo deQueiroz

Xerox Corporation

Webster, New York 14580

queiroz@wrc.xerox.corn

Dr. Eli Saber

Xerox Corporation

Webster, New York 14580

eli_saber@xn.xerox.com

\section{Publicity}

Mr. George Sotak

Eastman Kodak Company

Rochester, New York 14653

sotak@image.kodak.corn 


\section{PROGRAM}

8:20 Registration

8:40 Welcome and Introduction

\section{DOCUMENT PROCESSING}

\section{Session Chair: Andreas Savakis}

8:50 Adaptive Color Halftoning Using a Blue Noise Mask with Symmetric Error Compensation, 1 Q. Yu, K. Parker, University of Rochester

9:10- A Novel Printer Model Based on a Two-by-two Concept for Color Halftoning, S. Wang, 2 Xerox Corporation

9:30 Total Least Squares Regression in Color Printer Calibration, M. Xia, A.M. Tekalp, University of Rochester; E. Saber, G. Sharma, Xerox Corporation

9:50 A Systems Approach to Color Scanning, G. Sharma, Xerox Corporation 4

10:10 Approximate String/Segment Matching for Handwritten Word Recognition, J.T. Favata, 5 SUNY Buffalo

\section{COMPUTER VISION}

\section{Session Chair: A. Murat Tekalp}

10:40 Interactive Augmented Reality using Uncalibrated Cameras, J Vallino, University of Rochester

11:00 Clustering, Indexing and Retrieval in Image Databases, A. Zhang, G. Sheikholeslami, SUNY Buffalo

11:20 On Image Fusion for Concealed Weapons Detection, L. C. Ramac, M. K. Uner, P.K. Varshney, Syracuse University; M. Alford, Rome Laboratory

11:40 Segmentation of Phalangeal Bones in Hand Radiographs, T. Pawlicki, R. Gaborski, Eastman Kodak Company 


\section{APPLICATIONS}

\section{Session Chair: Ricardo de Queiroz}

1:00 Imaging the Dead Sea Scrolls, K. T. Knox, Xerox Corporation; R. H. Johnston,

R. L. Easton Jr, Rochester Institute of Technology

1:20 Evaluation of a the Effectiveness of a Neural Network Classifier in Locating Regions of Interest in Cervical Spine Radiographic Images, R. S. Gaborski, Eastman Kodak Company; S. McAleavey, K. Parker, University of Rochester

1:40 Automatic Detection of Human Eyes In Digital Images, J. Luo, Eastman Kodak Company

2:00 Generalized Basis Fringes for Digital Holography and Holovideo, J. Cozakas, E. Rusjan, D. Zimmermann, SUNY Institute of Technology at Utica

2:20 Jump Size Distribution Measurements in Microscopic Contact Line Motion, J. Marsh, SUNY Institute of Technology at Utica/Rome

\section{IMAGE AND VIDEO COMPRESSION \\ Session Chair: Gozde Bozdagi}

2:50 Active-Selective JPEG Coding, M. Ramos, S. S. Hemami, Cornell University 15

3:20 Processing JPEG-Compressed Images, R. de Queiroz, Xerox Corporation 17

3:30 Human Sensitivity to Quantization Errors in Image Subbands, S. S. Hemami, 18 Cornell University

3:50 Lossless Image Compression by Logic Minimization, D. Zhang, A. I. Cole, A. Albicki, 20 University of Rochester

4:10 2-D Triangular Mesh Geometry and Motion Compression, P.LL. van Beek, A.M. Tekalp, 21 University of Rochester

4:30 Multi-resolution Image Sequence Coding for Storage and Retrieval, D. Nadarajah, 23 Raghuveer Rao, Rochester Institute of Technology 


\title{
Adaptive Color Halftoning using a Blue Noise Mask with Symmetric Error Compensation
}

\author{
Qing Yu and Kevin J. Parker \\ Department of Electrical Engineering \\ University of Rochester \\ Rochester, NY 14627 \\ qiyu@ee.rochester.edu
}

Color halftoning using stochastic screens (or Blue Noise Masks) eliminates the screen rotation requirement and Moire artifacts that typically come with conventional clustered-dot screen scheme. In this paper, we will propose a color halftone scheme that improves halftone smoothness and takes color reproduction into account as well.

Typically, one blue noise mask and its inverted version will be used to halftone the cyan and magenta color channels, and the yellow channel is halftoned with symmetric error compensation in the CIELAB vector color space. We will show that :

1. The invert scheme makes the output color halftone dots, in this case cyan and magenta, smoothly dispersed, therefore reducing color interference noise and increasing spatial frequency at light tones for better visual quality.

2. By making yellow channel adaptive, we obtain increased flexibility to manipulate the output so as to reduce colorimetric error while permitting customization to specific printing hardware.

3. By using symmetric error Compensation instead of conventional vector error diffusion, the color smearing artifacts are greatly reduced.

The special mask design will be outlined and a discussion on symmetric error compensation scheme will also be given.

Keywords: color halftoning, stochastic screen, Blue Noise Mask, invert, symmetric error compensation, CIELAB. 


\title{
Total Least Squares Regression in Color Printer Calibration
}

\author{
Minghui Xia ${ }^{1}$, Eli Saber ${ }^{2}$, Gaurav Sharma' , and A. Murat Tekalp ${ }^{1}$ \\ ${ }^{\mathrm{I}}$ Department of Electrical Engineering \\ University of Rochester \\ Rochester, NY 14627 \\ ${ }^{2}$ Xerox Corporation \\ Webster, NY 14580
}

In halftone color printer calibration, Neugebauer based modeling plays an important role in obtaining device characterization profiles. In this paper, we propose the use of total least square (TLS) based regression techniques to estimate the appropriate parameters for various Neugebauer models. Compared with traditional least squares (LS) based techniques, TLS is physically more appropriate because it accounts for errors in the measured reflectances of both the selected primaries and the modeled reflectances. The TLS based techniques are tested on two color printers with random and doton-dot halftone screen configuration, and compared with the LS based algorithms. The results show that the TLS based algorithms yield a significant improvement over the $\mathbf{L S}$ based techniques in estimating the model parameters. 


\title{
A Systems Approach to Color Scanning
}

\author{
Gaurav Sharma \\ Digital Imaging Technology Center \\ MS 0128-27E \\ Xerox Corporation \\ Webster, NY 14580 \\ sharma@wrc.xerox.com
}

\begin{abstract}
A summary of recent research in applying a signal-processing systems approach to color scanning is presented. Human color vision and color scanners are described in a vector-space framework. The vector-space description is used to explain color recording capabilities of color scanners and to motivate criteria for their design. Additional considerations arising from nonlinearity of human color perception and from device noise are introduced and a comprehensive figure of merit for color scanners incorporating these factors is presented. The figure of merit is compared with several existing measures for scanner performance in terms of agreement with perceived color performance and computational requirements.
\end{abstract}




\title{
Approximate String/Segment Matching for Handwritten Word Recognition
}

\author{
John T. Favata \\ Cedar Research/SUNY Buffalo \\ 520 Lee Entrance, Suite 202 \\ Amherst, $\mathbf{N Y} 14228$ \\ favata@cs.buffalo.edu \\ http://www.cs.buffalo.edu/-favata
}

This work focuses on the problem of isolated off-line general word recognition using an approximate segment-string matching algorithm. Several recently proposed word recognition algorithms use the strategy of directly matching the word image stroke segments (with OCR estimates) to the character sequences in each of the lexicon words. This idea works very well under ideal conditions, however, many applications require the recognition of text in the presence of document noise, poor handwriting, and lexicon errors. These factors require careful design of the matching strategy so that a moderate amount of any form of degradation does not cause a recognition failure. A segment-to-string matching algorithm is proposed which will robustly recover from moderate levels of noise and system errors. This algorithm is developed in the context of a complete word recognition system (called the Character Model Word Recognizer (CMWR)) and serves as its final post-processing module.

Word recognition can be viewed as a constraint satisfaction problem. The most basic of these constraints are the plausible character shapes embedded in the strokes of the word. This, by itself, produces a large number of possible word interpretations (symbol identities) because of a certain level of ambiguity in handwritten characters. These interpretations of the word can be represented by an Augmented Directed - Graph, $G=(V, A)$ where $V$ is a set of nodes which represent the segmentation points of the word, and $A$ is a - set of edges which contain the estimates of the likelihood of the embedded characters. These estimates will be denoted as $D_{i}(S)$. We will label the elements of $V \mathbf{\infty}\left(s_{1}, s_{2}, \ldots s_{n}\right)$ where each $s_{j}$ is a segmentation point of the word. The elements of $\mathbf{V}$ are naturally ordered left to right with $s_{1}$ being the leftmost point of the word and $s_{n}$ being the rightmost point in the word. The argument $S$ to $D_{i}($.$) is a set \left\{s_{i} . s_{j}\right\} \quad(i<j)$ which represents all of the strokes between segmentation points $\mathrm{s}$, and $\mathrm{s}$, Let a word in the lexicon be made up of a sequence of characters, $\left\{C_{1}, C_{2}, \ldots, C_{n}\right\}$, the task is to find a path $P^{*}=\left\{A_{\pi 1}, A_{\pi 2}, \ldots . A \quad\right\}(m<=n)$ of edges, where $A=\left(\pi_{j}, \pi_{j+1}, \ldots, \pi_{k}\right)$ is a sequence of segmentation points $\left(\pi_{j}\right.$ is some left point and $\pi_{k}$ is a right point, $j<k$ ) that maximizes a likelihood (cost) function.

However, because of the general ambiguity of handwriting, system segmentation/OCR errors and other failures. it is necessary to estimate the best-cost path in $\mathrm{G}$ using approximation techniques. A promising technique uses a Beam Search algorithm The idea is to carry the L best partial matches forward by using a queue structure to hold the current match state. The algorithm iteratively takes each of the current best $\mathbf{L}$ partial matches and expands them to find the next incremental match between a character and a segment. Each new partial match is evaluated by the scoring function and the new best $\mathrm{k}$ paths are retained. Consider a partial match of char ${ }_{1}$ to char $r_{j}$ with $\operatorname{seg}_{1}$ to $\operatorname{seg}_{k}$, the following situations are likely to occur: (1) Normal March char ${ }_{j+1}$ matches $\left[\mathrm{seg}_{k+1} . . \mathrm{seg}_{k+1+i}\right.$ ], (2)Spurious Segments - $\left[\operatorname{seg}_{k+1} . . \operatorname{seg}_{k+1+i}\right]$ are noise, (3) Missing Segments - no segments correspond to char ${ }_{j+1}$, (4) OCR failure - segments present but OCR did not correctly identify underlying character. Several heuristics are introduced to compensate for these errors: Heuristic 1 - continue a path even if there is no OCR indication for this character, Heuristic 2 - assume a single segment is noise and Heuristic 3 - assume no segments exist for this character.

Results of the CMWR algorithm on standard sets of handwritten words are presented along with an analysis of the effectiveness of each of the heuristics.

1997 WNY Image Processing Workshop - 5 


\title{
Interactive Augmented Reality using Uncalibrated Cameras
}

\author{
James Vallino \\ Department of Computer Science \\ University of Rochester \\ Rochester, NY 14627 \\ vallino@cs.rochester.edu
}

Augmented reality is an area within virtual reality research whose goal is the seamless merging of elements from a synthetic virtual world with a view of the real world. The synthetic elements enhance the users perception of the real scene. Applications for this technology can be identified in many areas, including, medicine, equipment maintenance, and the military.

Correct rendering of virtual objects that are to be composited with the real scene is accomplished by having the synthetic camera in the computer graphics system mimic the projection operation of the scene viewer. Previous methods for augmenting reality have defined the operation of the scene viewer, such as a video camera, in a standard 3D Euclidean reference frame. Providing the metric information necessary for the synthetic camera to mimic this operation requires careful calibration of elements in the real world, knowledge of the calibration parameters of the camera, and the position of the person interacting with the system. Each one of these inputs introduces a source of error in the system.

Motivated by work in computer vision in uncalibrated environments (Koenderink and van Doom 1991) a new method for obtaining image registration in an augmented reality system has been developed. Assuming an affine camera model, the camera motion and the structure in the scene are represented in an affine frame which can be determined by analysis of video frames fram an uncalibrated camera. The method only requires the ability to track at least four rigid features through the video sequence. Scene structure is invariant to camera motion subject to the limitations of the affine model. In addition, the camera motion can be determined from the position of the tracked features in each video frame. In this affine coordinate system it is still possible to perform a number of operations that are required from a practical augmented reality system. These operations include: animation of the virtual objects, occlusion of a virtual object by a real object, and user interaction with virtual objects. The system includes a PHANToM force feedback haptic device. This allows the user to have touch sensations while interacting with virtual objects in the augmented view of the scene.

The presentation will show video tape sequences that demonstrate the real-time operation of an augmented reality system using uncalibrated cameras.

A general introduction to augmented reality and more details about the method of operating with uncalibrated cameras can be found at http://www.cs.rochester.edu/u/vallino/research/AR. Those web pages also have links to several MPEG video clips that demonstrate the system operation.

This work has been performed under the supervision of Profs. Chris Brown and Kiriakos Kutulakos.

Koenderink, J. J. and A. J. van Doom (1991). Affine Structure from Motion. Journal of the Optical Society of America A 8(2): 377-385. 


\title{
Clustering, Indexing and Retrieval in Image Databases*
}

\author{
Aidong Zhang and G. Sheikholeslami \\ Department of Computer Science \\ State University of New York at Buffalo \\ Buffalo, NY 14260
}

In most existing content-based retrieval approaches for image databases, significant features must first be extracted from images in their pixel format. The features are numerical measurements to characterize an image. These features are then used to index the images for efficient retrieval. With the large volume of data stored in an image database, efficient retrieval would be specially critical. Methods have been developed for indexing and accessing alpha-numerical data in traditional databases. However, the traditional approaches to indexing may not be appropriate in the context of content-based image retrieval. In this context, a challenging problem arises with many image databases, within which queries axe posed via visual or pictorial examples (termed visual queries). A typical visual query might entail the location of all images in a database that contain a subimage similar to a given query image.

In most existing indexing techniques, feature vectors of images are used to distinguish images through measuring distances between feature vectors. Our experimental results demonstrate that this type of approaches can be effectively used to perform content-based retrieval based on similarity comparison between images. However, we encounter a critical problem that the feature vectors of some semantically irrelevant images may be located very close in the feature space. For example, two images with water and stone respectively are semantically not similar, but the distance between their feature vectors in the feature space is very small. Given a query such that its feature vector is located in the neighborhood of the feature vectors of the two given images, both images are highly possible to be retrieved together in response to the query. Thus, indexing itself based on the closeness of feature vectors in the feature space sometimes may not provide satisfiable solutions. An effective clustering approach needs to be integrated into indexing techniques for efficient retrieval of image data. Once the query is narrowed to a specific category, image retrieval can then proceed efficiently.

In this presentation, we will discuss effective image data representation and organization approaches for large-scale image databases. An effective block-oriented image decomposition structure is used as a fundamental data model for representing image content. A clustering and indexing mechanism is then proposed to categorize images based on feature similarity. Efficient image retrieval is supported. Experimental analysis are conducted and presented to demonstrate the effectiveness and efficiency of the approaches.

- This research is supported by Xerox Corporation. 


\title{
On Image Fusion for Concealed Weapons Detection
}

\author{
L. C. $\operatorname{Ramac}^{1}$, M. K. Uner ${ }^{1}$, P. K. Varshney ${ }^{1}$, and M. Alford ${ }^{2}$ \\ ${ }^{1}$ Syracuse University \\ Syracuse, NY 13244 \\ e-mail: varshney@cat.syr.edu \\ ${ }^{2}$ Rome Laboratory \\ Griffiss AFB, NY 13441
}

When viewing a scene for an object recognition task, one imaging sensor may not provide all the information needed for the job. One way to obtain more information is by using multiple sensors. These sensors should provide images which contain complementary information about the same scene. Then we use image fusion to combine the information from the different source images.

The image fusion process consists of decomposing the individual source images into multiresolution representations. One type of multiresolution representation is based on the wavelet transform. Once the source images are decomposed, they are combined using some feature selection algorithm. This method allows features at different levels to be combined independently so that all important details are maintained in the final composite image. The feature selection algorithms vary according to what information is needed for a specific task. The combination at this stage results in a composite multiresolution representation which is then reconstructed to form the final image. After fusion, the final composite image contains the important information taken from the individual source images.

We are developing image fusion algorithms for concealed weapon detection (CWD) applications. Fusion is useful in situations where the weapons are not completely visible from individual sensors. This may include cases where the individual sensors are viewing from different positions or cases where the sensor types are different, e.g., IR and MMW sensors. Fusing these types of images results in composite images which contain more complete information for CWD applications such as detection of concealed weapons on a person. In this talk we will present our most recent results in this area. 


\title{
Segmentation of Phalangeal Bones in Hand Radiographs
}

\author{
Ted Pawlicki and Roger Gaborski \\ Eastman Kodak Company \\ Rochester, NY 14650
}

The study of bone mass and its changes due to age, various diseases and metabolic disorders is of primary importance for medical diagnosis and treatment. Radiographic absorptiometry (RA), or photodensitometry, is a sensitive, noninvasive, quantitative method used to assess bone mineral changes. The phalanx bones seen in dorso-palmar hand radiographs are often used for RA measurement. Historically, the intervention of a human operator is required to segment these bones prior to quantitative measurement. This talk presents a system for the automation of such segmentation. 


\title{
Imaging the Dead Sea Scrolls
}

\author{
Keith T. Knox ${ }^{1}$, Robert H. Johnston' , and Roger L. Easton Jr. ${ }^{2}$ \\ ${ }^{\mathrm{I}}$ Xerox Corporation \\ Webster, NY 14580 \\ ${ }^{2}$ Center for Imaging Science \\ Rochester Institute of Technology \\ Rochester, NY 14627
}

Digitally scanned photographs of Dead Sea Scrolls have been enhanced to increase the legibility of degraded text in these 2000-year-old documents. This paper describes the enhancement of text in the scrolls using a linear color space to separate the text from the degraded background. Initial results reveal characters whose existence were not previously known to the scholars studying these scrolls. 


\title{
Evaluation of the Effectiveness of a Neural Network Classifier in Locating Regions of Interest in Cervical Spine Radiographic Images
}

\author{
Roger S. Gaborski ${ }^{1}$, Steve McAleavey ${ }^{2}$, and Kevin Parker ${ }^{2}$ \\ ${ }^{1}$ Eastman Kodak Company \\ Rochester, NY 14653-5740 \\ ${ }^{2}$ Department of Electrical Engineering \\ University of Rochester \\ Rochester, NY 14627
}

Locating regions of interest in medical radiographic images is important for optimal rendering and computer-aided diagnosis. In the work reported here two techniques are compared, a matched filter and a neural network classifier.

A set of ten cervical spine radiographic images were selected. Five of the images were used for training, the remaining five for testing. A dynamic compression technique was used as an image enhancement preprocessing procedure to equalize the appearance of the images.

The matched filter technique used a bank of approximately 100 gray scale templates that were derived from the training set. The templates were based on a single scaled and rotated vertebrae. The scale varied over a factor of two and the rotation over $\mathbf{4 5}$ degrees. In testing, the vertebrae were localized by correlating the test images with each template, generating 100 correlation images. Within each correlation image, peaks were selected as possible location of the vertebrae. A suppression technique was used to resolve differences between the different correlation images.

A neural network classifier was developed which classified 24 by 30 pixel regions as either containing a vertebrae or not. The network had $\mathbf{7 2 0}$ input neurons, with two hidden layers, composed of 16 neurons and $\mathbf{8}$ neurons. The output layer had two neurons. Initially, 1000 samples were selected from the training set representing the vertebrae and non-vertebrae data. The network was trained and tested on the training data. Misclassifications were collected and used in a bootstrap manner to increase the training set to $\mathbf{2 0 0 0}$ samples. The network was then retrained and tested on the testing image set.

A priori knowledge of the anatomical structure of the cervical spine and a mask based on background and foreground detection were used to reduce the number of false positives within each image for the matched filter approach and the neural network classifier. 


\title{
Automatic Detection of Human Eyes In Digital Images
}

\author{
Jiebo Luo \\ Imaging Science Division \\ Eastman Kodak Company \\ 1/65/RL, MC 01816 \\ Rochester, NY 14650 \\ luo@image.kodak.com
}

A method for automatic eye detection in consumer-type imagery is developed. This method generally consists of three stages: pre-screening, template matching and verification using hypothesis reasoning. First, flesh regions in the image can be detected if color balance is reliable. From this data, the approximate scale and tilt of the potential face are estimated. Also in the pre-screening stage, valley regions in the intensity image are identified to eliminate unlikely regions from further consideration. Second, using a generic eye template with zone partition, a cross-correlation is computed at the locations determined by the pre-screening process. Finally, hypotheses of eye pairs are formulated and evaluated using a set of figures of merit. The eye locations are determined by the winning pair within each selected (flesh) region.

The proposed eye location algorithm provides automation, precision, reproducibility and consistency. This eye location algorithm has been adapted to support several specific applications. In KODAK DIGITAL SCIENCE (TM) Image Verification System (IVS), a semi-automatic version of the eye location algorithm has been used to perform the required spatial normalization of face images in order to achieve efficient data compression. 


\title{
Generalized Basis Fringes for Digital Holography and Holovideo
}

\author{
Jason Cozakos, Edmond Rusjan, and Derrik Zimmermann \\ SUNY Institute of Technology \\ Utica, NY 13504-3050 \\ edmond@sunyit.edu
}

Digital holography and holovideo are young and rapidly developing technologies which promise to bring us true three dimensional images and movies. Future applications include three dimensional displays in medical imaging (MRI, CAT), scientific visualization, engineering (CAD), virtual reality, and telecommunications (3DTV), Recent improvements in the computational algorithms, specifically the diffraction specific method, have led to first interactive three dimensional holographic images. Once the technology is established it will require the reimplementation, if not reinvention, of the standard image processing algorithms.

The diffraction specific method synthesizes the hologram from many basis fringes. Basis fringes are localized to a small area of the hologram, the so-called hogel, and focus the light emanating from the hogel into a beam pointing into a specified direction. Basis fringes can be precomputed and then used as a building block for generating holograms resulting in great savings in time, High quality of basis fringes is crucial for the quality of the synthesized hologram.

In this paper we propose the concept of a generalized basis fringe (GBF), i.e., a fringe which is localized both in space domain and in frequency domain, and has a continuous profile, as opposed to the basis fringes with rectangular profiles studied so far. We compute triangular, trapezoidal, quadratic and Gaussian GBF's using two methods. The first method starts with a random fringe, applies the spatial constraints, Fourier transforms the fringe, applies the spectral constraints, transforms back to the space domain, and iterates this process until it converges. The second method is of the least squares type and minimizes the sum of the errors in the spatial and in the spectral domain. We compare the quality of holograms synthesized from GBF's of various profiles in order to determine the best fringe profile.

We use the GBF technology to make the first attempts at holographic image processing. In particular, we develop an algorithm for edge enhancement based on interpolating between different sets of GBF's. Some of the holograms will be shown at the workshop. 


\title{
Jump Size Distribution Measurements in Microscopic Contact Line Motion
}

\author{
John Marsh \\ Assistant Professor of Physics, Arts and Sciences, \\ SUNY Institute of Technology at Utica/Rome \\ Utica, NY 13504-3050
}

The author presents details of an algorithm used to extract jump size distributions from video of microscopic contact line motions across a heterogeneous solid surface. This is one aspect of a project investigating the connection between microscopic contact line (CL) motion and macroscopic contact angle hysteresis (CAH). The CL is simply the edge of a meniscus where a fluid/fluid interface intersects a solid surface. $\mathrm{CAH}$ is the existence of a range of stable static contact angles (falling between the advancing and receding contact angles), and is associated with microscopic metastable states of the CL. In an attempt to quantify this connection, solid surfaces with controlled surface heterogeneity (patterned monolayers of alkanethiol on gold) are microfabricated and quasi-statically withdrawn from a bath of liquid. The CL is imaged under low magnification (field of view: $1 \mathrm{~mm}$ ) as it recedes over the solid surface. The video clearly shows the stick-slip motion as the CL recedes over heterogeneities on the solid surface. The algorithm automatically extracts the jump size distributions from the digitized sequences of images, and has been applied to sequences of 720 image frames, covering a time span of 6 minutes (corresponding to a contact line motion over approximately $1 \mathrm{~cm}^{2}$ ). Each image has a mostly horizontal, high contrast edge, allowing easy extraction of the CL shape. The differences between the CL shapes in adjacent frames are then taken and used to form an 8 bit secondary image, whose gray levels indicate the CL speed. Gray levels below 128 indicate a negative CL velocity (stick) whereas gray levels above 128 indicate a positive CL velocity (slip). The stick/slip process is clearly visible in the secondary image as corresponding pairs of dark/light regions. The secondary image is thresholded to highlight those portions corresponding to slip, and a particle counting algorithm is implemented, counting individual CL jumps. The final jump size (in square microns) is calculated from the known frame rate and the average gray level (i.e. velocity) of the pixels in the particle (i.e. jump). Issues in implementation, as well as a demonstration of the technique will be presented. 


\title{
Activity-Selective JPEG Coding
}

\author{
Marcia G. Ramos and Sheila S. Hemami \\ Visual Communications Lab \\ Cornell University \\ Ithaca, NY 14853 \\ \{mramos, hemami\}@ee.comell.edu
}

This paper presents a modified JPEG algorithm that provides higher visual quality than the $Q$-factor scaling method commonly used with JPEG implementations by considering the different activity regions of an image. Psychophysics studies reveal that there are three activity regions of distinctive perceptual significance to human observers: strong edges, smooth regions, and textured or detailed regions. Since the perceptual quality of compressed images is ultimately evaluated by humans, image compression algorithms can take advantage of the different human visual system (HVS) perceptual responses to each region to provide images with the best visual quality possible at all bit rates. The activity-selective JPEG algorithm proposed here is based on a multiresolution image segmentation technique that identifies the edge, smooth, and detailed regions of the image. The activity classifications are used to adapt the DCT quantization step sizes to the activity of the block.

The multiresolution image segmentation technique identifies the three activity regions of an image by using only the $\boldsymbol{L} \boldsymbol{H}$ and $H L$ high frequency subbands of a 3-level hierarchical subband decomposition. The image is divided into $8 \times 8$ blocks, and each block is classified into one of the activity regions by examining the high frequency coefficient decay across the scales of the subband decomposition based on the Holder regularity property of wavelet coefficients. The wavelet coefficients that correspond to smooth areas decrease rapidly as the resolution varies from coarse to fine. A slow coefficient decay indicates the presence of activity in the image, corresponding to an edge or a detailed area. In particular, strong edges are indicated by isolated features correlated over several scales while the behavior of the coefficients in detailed areas appears random and exhibits no such correlation. The decay information is used to form an activity measure that is thresholded to yield the final classification criterion.

The activity classifications are used to adapt the quantization step sizes to the activity level of the block. For each 8x8 DCT block, the step sizes of the quantization matrix are scaled according to the perceptual region the block is in, i.e., smooth, edge, or detailed region. The DC coefficient is kept intact to avoid large blocking effects and to smooth out the errors across the entire image. This in contrast to the Q-factor approach used in most JPEG codec implementations, where the entire is scaled. After several tests on different images from the USC database, integer scalings ranging from 1 to 8 were adopted. Scales larger than 8 improve the compression ratios but lead to very distorted images. A scaling of 1 for all the segmented regions corresponds to the JPEG standard with the recommended matrices. For each image, all possible combinations of scalings for blocks belonging to one of the three perceptual components are simulated, and the rate-distortion curve is generated. At each bit rate, the scalings are chosen to maximize $\Delta R / \Delta D$. At high bit rates, all the segmented regions tend to have small scalings. As the rate decreases, blocks corresponding to detailed regions will have progressively higher scalings, followed by blocks in edge regions, and lastly, blocks in smooth regions. 
This results in a considerable perceptual improvement since the compression artifacts are distributed according to the HVS sensitivity to different acceptable distortion levels in the three activity regions.

A comparison of activity-selective JPEG and JPEG's $Q$-factor approach was done both subjectively and by comparing RD curves. In general, for a fixed distortion level, the rates are approximately equivalent. The images show that the blocking effects that are especially common in smooth regions of JPEG compressed images have been considerably reduced by using the segmentation technique. This has a strong visual impact in determining the perceptual quality of the compressed images, as it diminishes the distortion in the smooth areas of the image (most sensitive to noise as compared to the other two regions). In general, the activity-selective JPEG yields higher visual quality images at the same bit rates (slight overhead due to transmitting the locations of the smooth, edge, and detailed blocks, as well as the scalings used for each block) especially in the smooth regions and around the strong edges that appear adjacent to large flat areas of the image. For more detail on this research and to access images, please refer to http://foulard.ee.cornell.edu/marcialasjpeg.html. 


\title{
Processing PEG-Compressed Images
}

\author{
Ricardo de Queiroz \\ Xerox Corporation \\ 800 Phillips Rd, M/S 128-27E \\ Webster, NY 14580 \\ queiroz@wrc.xerox.com
}

Image compression became a necessity when dealing with large images (or several smaller images) to save space and increase efficiency. Typical lossless (or reversible) coders can barely attain a compression ratio of 2:1 for most images. Thus, users often cope with lossy algorithms, which tend to slowly degrade the image quality in exchange for more aggressive compression ratios. JPEG is a widely adopted compression standard on which we base our discussion. For simplicity we concentrate on the most popular mode, known as baseline JPEG, although several results may also apply to other modes of operation.

The objective of the paper is to present techniques that allow the processing of JPEG-compressed data without decompressing it, i.e. Operations are performed in the "JPEG-compressed" domain. The meaning of "JPEG-compressed" domain also deserves some clarification. JPEG compression is performed by a series of operations: transform, quantization, zigzag scanning, DPCM, and entropy coding. Decompression is accomplished by performing inverse steps in an inverse order. We assume the data is only available in compressed format. Therefore, the first operations to be applied to the data are part of the decompression routine, and an effort is made to perform as few operations as possible. In some cases, only partial entropy decoding is needed, while in others we use most of the data in transform domain.

In most operations we discuss (outputs are also JPEG-compressed images) we apply the following steps: partial block decompression, fast processing, partial block compression. Each operation described in this paper can be alternatively implemented in the trivial way: decompressing, processing, and recompressing the image. The motivation for processing compressed images is because it saves memory and/or improves speed.

We will present in more details some algorithms for processing images in compresseddomain. Apart from the obvious methods (that simply explore the fact that DCT is a linear transform and that by modifying the DC coefficient one can modify the average luminance of the image), we present a set of fast algorithms to resize, preview, rotate, mirror, manipulate (e.g. cut and paste), segment, color correct and recompress a JPEG-compressed buffer. 


\title{
Human Sensitivity to Quantization Error in Image Subbands
}

\author{
Sheila S. Hemami \\ Visual Communications Lab \\ School of Electrical Engineering \\ Cornell University \\ Ithaca, NY 14853
}

Perceptual thresholds for wavelet quantization error to date have been proposed based on tests of sensitivity to individual basis functions [1], with quantization step sizes that are independent of individual band energy or interband energy distributions. We performed a large-scale study of human sensitivity to of quantization noise in individual high-frequency subbands within images having differing characteristics (edge orientations, depth-of-field, relative subject size). The test was designed with the guidance of a psychologist and performed on a World-Wide-Web platform, to encourage the participation of non-experts and to gather results from viewing environments in which compressed images are most typically viewed. The results are used to create a modified bit allocation algorithm that takes perceptual sensitivites into account.

Two luminance-only images of size $256 \times 256$ were presented in the psychovisual test. This size was selected as being typical for internet image exchange in a reasonable amount of time, and therefore commonly encountered. The image selection criteria included the presence of edges in various directions, the contrast, and the depth-of-field. The 7-9 biorthogonal filters were used in a 2-level hierarchical wavelet decomposition. Assuming an average viewing distance of eighteen inches and a 72-100 dots-per-inch monitor resolution, the first level of subbands contain information to a maximum spatial frequency of approximately 16 cycles/degree and the second level contains information to maximum spatial frequencies of 8 cycles/degree.

Seventy trial images were generated for each of the two test images, with 10 images for the lowfrequency band and each of the 6 high-frequency subbands. In each set of 10 images for a single subband in a single test image, the subband was uniformly quantized with step sizes given by (dynamic_range)* $\mathrm{k} / 9$ where $\mathrm{k}=0$ corresponds to no quantization and $\mathrm{k}=9$ corresponds to setting the entire subband to 0 . The tests measured absolute thresholds in both an ascending and descending series. Subjects were asked to increase (decrease) the quantization step sizes in the ascending (descending) series until the image quality just began to degrade (become acceptable). The results of the two series are averaged and the resulting step sizes are called the "just-noticeable-difference step sizes" (JNDSS). These sizes are then normalized with respect to the standard deviation of the subbands and referred to as the normalized JND step sizes (NJNDSS).

Several general inter- and intra-scale sensitivity relationships emerge in the results. The NJNDSS for the lowest-frequency band for both images are nearly identical. As expected, sensitivity to errors in the HH bands is much lower than sensitivity to errors in the LH and HL bands, and both the JNDSS and NJNDSS are lower for the coarser subbands. Visual masking clearly occurs as a function of the energy distribution among bands at the same scale, as both the JNDSS and NJNDSS are not equal for the LH and HL bands at the same scale. 
Because sensitivity to each subband was tested independently, the obtained JNDSS and NJNDSS are upper bounds for quantization step sizes. In fact, if the resulting step sizes are used simultaneously, they must be halved to produce an acceptable image. We note that while the resulting images exhibit artifacts seen in the individually selected images from the test, they appear unacceptable to "image processing experts." We conclude that non-experts using a blind test have a much higher tolerance for errors than we expect.

Using the results directly to develop a psychovisual quantization strategy is difficult because the results suggest a single set of quantization step sizes. However, for a fixed orientation the ratio (band variance/NJNDSS) (level 2) / (band variance/NJNDSS) (level 1) is constant, and in fact is equal to the same constant, regardless of orientation. This ratio and the three orientations can be used to include three additional constraints in the standard bit allocation solution. Images quantized with the resulting step sizes exhibit artifacts identical to those quantized with the actual step sizes from the test, and can be thought of as existing on a "psychovisual ratedistortion curve."

[1] A. B. Watson et. al., "Visual thresholds for wavelet quantization error," IEEE Trans. Image Processing, Vol. 6, No. 8, August 1997, pp. 1164-75. 


\title{
Lossless Image Compression by Logic Minimization
}

\author{
Daofa Zhang, Agnim I. Cole, and Alexander Albicki \\ Department of Electrical Engineering \\ University of Rochester \\ Rochester, NY 14627 \\ lulik@ee.rochester.edu
}

There are two types of compression: lossless compression and lossy compression. To losslessly compress images, we typically would use a popular variable length coding scheme to represent more frequently occurring patterns as shorter code words. The most popular lossless techniques are Huffman Coding, LZW Compression, Arithmetic Coding and Run-Length Coding.

In this paper, we explore logic minimization techniques to losslessly compress bi-level, and grayscale images. Each pixel of an image is regarded as a minterm of a logic function. By applying the logic minimization technique, we can reduce the number of cubes that represent that function. In this way, the number of terms that represent the image is minimized.

The algorithm we have developed proceeds as follows: an image is split into windows of fixed sizes $2 \mathrm{M} \times 2 \mathrm{~N}$ like $4 \times 4,8 \times 4,8 \times 8,16 \times 16$, or $32 \times 32$ for example. Then, using a popular two-level logic minimizer, ESPRESSO, these windows are minimized. The resulting cubes of the length $m+n$ consist of ones, zeros, and don't cares. They are either Huffman encoded, or encoded as fixed length vectors suitable for further compression. The latter resulted in better compression in our case studies.

We have investigated:

Compression ratios obtained for differing window sizes. The $8 \times 8,4 \times 8$, and $8 \times 16$ windows had the highest compression ratios of all the images. Optimum sizing depended heavily on an image's level of detail and window orientation (i.e. either $4 \times 8$ or $8 \times 4$ ). Larger windows dividing simple images gave a maximum compression ratio.

For 256 tone gray scale images, we separated all eight bits of each pixel into separate planes of bilevel images. By Gray encoding of gray scale pixels, we decreased the gradient between adjacent pixels and thus obtained fewer cubes in minimized logic functions.

We have compared our results to several popular lossless compression algorithms like UNIX pack [Huffman Coding] and compress. [LZW Coding]. Our technique, although more time consuming, outperformed them for almost every image in at least

a dozen images that we have tested.

Logic Minimization Compression technique is effective for long term storage of images where asymmetric compression time is desirable. We believe it to be especially suited for archiving medical imagery. The final results of our experiments including the comparison of our method with JBIG technique will be presented at the Conference.

J. Augustine, J. Jacob, and W. Feng, "Logic Minimization Based Approach for Compressing Image Data", 8th International Conference on VLSI Design, Bombay, India, January 1995. 


\title{
2-D Triangular Mesh Geometry and Motion Compression
}

\author{
Peter J. L. van Beek and A. Murat Tekalp \\ Department of Electrical Engineering and Center for Electronic Imaging Systems \\ University of Rochester \\ Rochester, NY 14627-0126
}

\section{Introduction}

Object-based video coding is concerned with efficient storage and transmission of digital video object planes instead of digital videoframes. We have recently proposed a mesh-based representation of video object planes which offers efficient video compression and manipulation functionalities [1] such as video interpolation and animation. In this method, a 2D triangular mesh is designed on the first object plane, as illustrated in Figure 1. The vertices of the triangular patches are referred to as the node points. The node points of the initial mesh are then tracked forward from object plane to object plane. Therefore, the 2D motion of an object is compactly represented by the motion vectors of the node points in the mesh. Furthermore, the 2D shape of a video object plane is compactly represented by the node points on the boundary of the mesh. Motion compensation can be achieved by texture mapping the patches from object plane to object plane according to respective node point motion vectors. This paper describes methods for compression of 2-D mesh geometry and motion and presents experimental results.

\section{Mesh Geometry and Motion Compression}

In our work, the 2D triangular meshes are constrained to be Delaunav. The Delaunay triangulation can be computed by both encoder and decoder, given the node points; thus, only the node point positions $\vec{p}_{a}=\left(x_{n}, \mathrm{y}\right.$, $)$ need to be encoded. To encode the node positions, the nodes are traversed one by one using a nearest neighbor search (see Figure 2a) and each $x$ - and $y$ - coordinate of a node is encoded differentially using the position of the previously encoded node as a predictor. Variable length coding is used to compress the coordinatedifferences.

Each node point $\vec{p}_{n}$ of a 2D mesh, moving from object plane $\mathbf{k}$ to plane $\mathbf{k}+1$, has a $2 \mathrm{D}$ motion vector $\bar{v}_{n}$. To encode the motion vector of a node point $\mathfrak{f i}_{n}$ that is part of a triangle $t,=\left\langle\overline{\vec{p}}_{l}, \overline{\vec{p}}_{m}, \vec{p}_{n}\right\rangle$, one can use the two motion vectors $\vec{v}_{l}$ and $\vec{v}_{m}$ of the two nodes $\vec{p}_{l}$ and $\vec{p}_{m}$ to predict $\vec{v}_{n}$. Note that if there is an initial triangle $t_{k}$ of which all three node motion vectors have been encoded, there must be at least one other, neighboring, triangle $t_{w}$ that has two nodes in common with $t_{k}$. Since the motion vectors of the two nodes that $\mathrm{t}$, and $t_{w}$ have in common have already been encoded, one can use these two motion vectors to predict the motion vector of the third node in $\boldsymbol{t}_{w}$. The actual prediction vector $\vec{W}_{n}$ is computed by averaging the two prediction motion vectors; the components of the prediction error vector $\vec{v}_{n}-\vec{w}_{n}$ are encoded by variable length coding. Then, other neighboring triangles of those triangles can be found, etc., until all triangles in the mesh have been processed (see Figure $2 b$ ).

\section{Experimental Results}

We report results on compression of the mesh geometry and motion. We evaluated the performance of our mesh geometry compression algorithm by comparing it to the compression performance of gzip on four different 2D meshes, see Table I. We evaluated the performance of our mesh motion compression algorithm (based on node motion vector coding) by comparing it to coding of a sequence of meshes one by one (using the mesh geometry compression algorithm) on three different mesh sequences, see Table 2. The mesh data was obtained by automatic mesh design and tracking as described in [I]. These results show that our mesh geometry compression algorithm far outperforms a standard compression algorithm such as gzip: furthermore, that our mesh motion vector compression algorithm outperforms compression of a sequence of mesh geometries. 


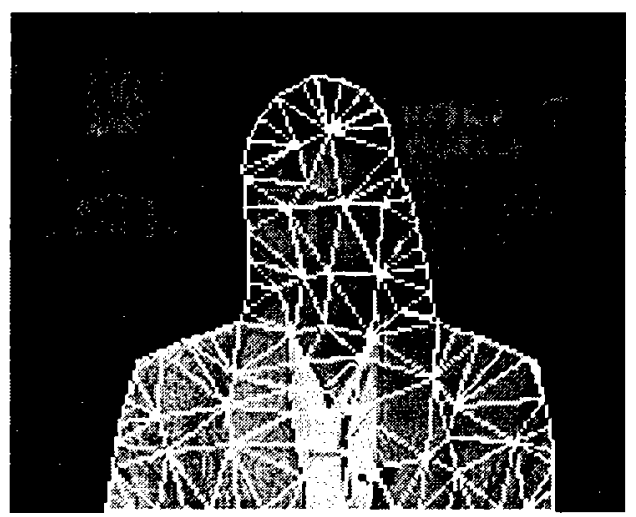

Figure I. Illustration of the object-based mesh modeling concept on the Akiyo video.

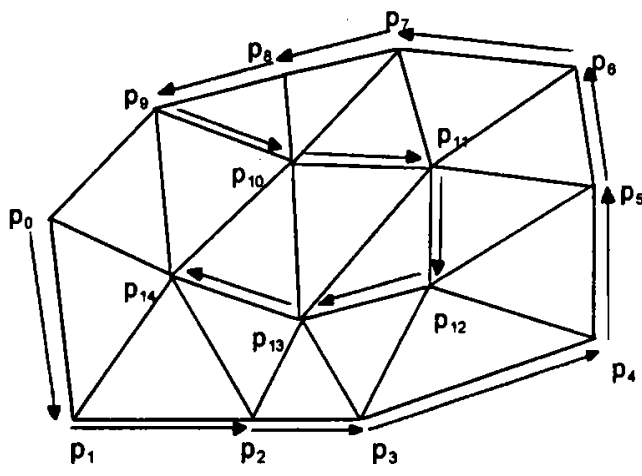

(a)

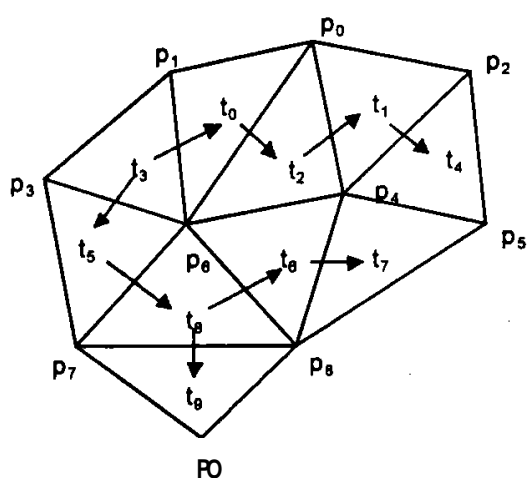

(b)

Figure 2. (a) Traversal of node points of a 20 triangularmeshfor node points location coding. Firstly, the boundary nodes are visited according to connectivity; then, the interior nodes are visited according to proximity. (b) Breadth-first traversal of triangles of a 20 triangularmeshfor node motion vector coding. Starting froman initial triangle at the top left of the mesh, triangles are visiting one by one, while node motion vectors are coded predictively.

\begin{tabular}{|l|r|r|r|}
\hline Mesh & ASCII file & gzip & proposed algorithm \\
\hline akiyo & 47568 & 18720 & 2683 \\
sean & 42584 & 16896 & 2494 \\
bream & 33960 & 13712 & 2156 \\
cyclamen & 226928 & 86840 & 12298 \\
\hline
\end{tabular}

Table I. Results of geometry compression of 20 Delaunay meshes. All numbers are bits.

\begin{tabular}{|l|r|r|}
\hline Mesh & Intra & Inter \\
\hline akiyo & 304508 & 56144 \\
sean & 277088 & 66585 \\
bream & 62420 & 30437 \\
\hline
\end{tabular}

Table 2. Results of combined 20 mesh geometry and motion vector compression (Inter)vs. compression of sequences of 20 meshes by geometry (Intra). 


\title{
Multi-resolution Image Sequence Coding for Storage and Retrieval
}

\author{
Dinesh Nadarajah and Raghuveer Rao \\ Department of Electrical Engineering \\ Rochester Institute of Technology \\ Rochester, NY 14623 \\ dxn8594@rit.edu,mrreee@ rit.edu
}

This paper discusses a technique to compress digital image sequences using multi-resolution techniques and is ideal for video storage and retrieval systems. The image sequence is arranged into Group of Frames (GOF) along the time axis. Critical subband decomposition is applied to each GOF. The number of frames in each group is determined by the subband filter to be used. Subband decomposition results in a low frequency frame and detail frame(s). The first GOF can be compressed using standard intra-frame compression methods. Subsequent groups are inter-frame coded. Motion estimation is performed on adjacent low frequency frames only. The detail and the prediction error frames are optimally quantized and entropy coded. A macro-block method (as used in MPEG) is adopted to code the error and detail frames, i.e. only macro-blocks with energy greater than a threshold are coded for storage and transmission. Since the detail and prediction error frames contain mostly high frequency information, spatial domain coding is preferred over transform coding. By controlling parameters such as number of frames in each GOF, quantization and motion estimation very good compression ratios can be obtained for reasonable quality video.

The technique lends itself very well to trick mode operations. Content based search can be performed on the low frequency frames, without the need for reconstruction of individual frames. Skipping of detail frame in trick mode operations also makes access faster. This method is low in complexity and thus can be easily implemented for on-line operations. 